\title{
Mechanisms of Heat Transfer for Axisymmetric Bubble Impingement and Rebound
}

\author{
D.B. Donoghue • A. Albadawi - Y.M.C. Delauré • A.J. Robinson • D.B. \\ Murray
}

Received: date / Accepted: date

\begin{abstract}
Heat transfer enhancement resulting from the impingement and rebound of bubbles in confined geometries can play an important role in heat transfer applications. Limited studies exist on the impact behaviour of large ellipsoidal bubbles against a horizontal surface, while the associated fluid flow field has received even less recognition. To address this, the current study investigates the dynamics of a single large ellipsoidal bubble impinging on a horizontal heated surface.

The bouncing dynamics have been explored by utilising synchronised high- speed and IR photography. Due to the large bubble size in the present study only a bubble with a low release to surface distance was found to have a symmetric bouncing event. The results showed that separated wake structures initially cooled the surface before the wake structures become counter productive and convect warm fluid onto the previously cooled surface. Two cooling zones were observed; the inner region due to the bubble and the outer region due to the bubble's wake.
\end{abstract}

Keywords Rising bubble . Bouncing bubble . Heat transfer enhancement · IR thermography

\section{Introduction}

Two phase flows occur extensively in nature and in technology and are utilized in systems ranging from healthcare to the energy industry. In particular, two

D.B. Donoghue

Department of Mechanical \&

Manufacturing Engineering,

Trinity College Dublin,

Ireland,

E-mail: donoghdb@tcd.ie phase flow plays an important role in convective cooling and chemical engineering applications ranging from complex cooling systems to mixing in reactors. Research has shown that two phase flows can produce exceedingly high heat transfer coefficients, which have the ability to be an order of magnitude higher than their single phase counterparts. This has motivated numerous investigations over the past century [1-9]. Although the dynamics of free rising bubbles have been studied extensively [10-14], research into their effects on heat transfer is limited; even fewer studies have been performed in relation to bouncing bubbles. A number of authors have investigated the effect of a bubble bouncing against a solid surface [15-19] under adiabatic conditions, but the corresponding heat transfer processes have received limited attention to date $[3,4,20,21]$.

Qiu \& Dhir [5] conducted an experimental study on the flow patterns and heat transfer associated with a vapour bubble sliding on a downward facing heated surface. They showed that the sliding bubble wake structure to the rear of the bubble enhanced heat transfer by introducing cooler liquid from the bulk to the surface. This was supported by the IR thermography experiments of Donnelly et al. [22] and more recently O'Reilly Meehan et al. [23] for a single air bubble and an in-line bubble pair, respectively. Complex "packets" of surface convective heat transfer enhancement were observed that was consistent with the vortex structures measured by O'Reilly Meehan et al. [24] using particle image velocimetry. Manickam \& Dhir [9] performed a further study, with the same experimental apparatus as Qiu \& Dhir [5]. Holographic interferometry was used to measure the temperature field surrounding the bubble. It was found that the bubble shape changed from a spheroid to an elongated cylinder and to a segment of 


\begin{tabular}{|llll|}
\hline \multicolumn{2}{|l|}{ Nomenclature } & & \\
$A_{c s}$ & cross sectional area $\left(\mathrm{m}^{2}\right)$ & $t$ & time $(\mathrm{s})$ \\
$C$ & specific heat capacity $(\mathrm{J} / \mathrm{kgK})$ & $\alpha$ & thermal diffusivity $\left(\mathrm{m}^{2} / \mathrm{s}\right)$ \\
$I$ & Amperage $(\mathrm{A})$ & $\delta$ & surface thickness $(\mathrm{m})$ \\
$R$ & resistance $(\mathrm{ohm})$ & $\kappa$ & thermal conductivity $(\mathrm{W} / \mathrm{mK})$ \\
$S_{x, y, z}$ & coordinate $(\mathrm{m})$ & $\rho$ & density $\left(\mathrm{kg} / \mathrm{m}^{3}\right)$ \\
$T$ & temperature $(\mathrm{K})$ & $\infty$ & bulk fluid conditions \\
$T_{m}$ & dimensionless temperature $(\mathrm{Eq} .4)$ & $f$ & foil \\
$d_{e}$ & equivalent diameter $(\mathrm{m})$ & $p$ & paint \\
$d x$ & camera pixel size $(\mathrm{m})$ & $s l$ & slice distance \\
$l$ & foil length $(\mathrm{m})$ & $s$ & test surface \\
$q^{\prime \prime}$ & heat flux $\left(\mathrm{W} / \mathrm{m}^{2}\right)$ & $x, y, z$ & coordinate direction \\
& & & \\
\hline
\end{tabular}

a sphere in that order, as the bubble slid on the heated surface.

Another study by Delauré et al. [6] utilised PIV to study local fluid velocities adjacent to a vertical and inclined heated surface along with the associated heat transfer. The swirling flow resulting from the shedding of vortices and fluctuations in the external fluid temperature due to bubble agitation were found to enhance the local heat transfer. The shedding of hairpin vortices from the bubble wake increased the temperature fluctuations in the thermal boundary layer by bringing colder fluid into contact with the heated surface.

Donnelly et al. [7] investigated the dynamics of a rising bubble and its influence on heat transfer from a vertical heated plate utilising the hot film sensor technique. Two methods of heat transfer enhancement were found, with the first being the bubble acting as a bluff body when its path was sufficiently close to the surface. However, when the bubble was further from the plate, the ensuing wake was found to affect heat transfer, although this was dependent on the path orientation of the bubble. A further study by Donoghue et al. [8] demonstrated the effect on heat transfer of whether the bubble's zig-zag path is normal or parallel to the heated surface, with the zig-zag path being controlled by the nozzle geometry.

With regard to bubble impact on horizontal surfaces, a recent study by Donoghue et al. [25] investigated coupled convective heat transfer and bubble motion due to the effect of a single $3.4 \mathrm{~mm}$ air bubble rising through water and impacting and bouncing on a heated horizontal surface. The time varying convective heat flux distribution was quantified for a single bubble impact and bouncing event and the detailed results were explained in terms of the bubble dynamics.

To date limited studies have been presented which illustrate the complex relationship between bubble motion and associated heat transfer from a surface. To address this, simultaneous measurement of heat transfer, fluid motion and bubble motion must be performed in order to better understand these complex interactions. The focus of this study is on a large, axisymmetric air bubble rising through water and impacting on a heated horizontal surface. The time varying convective heat flux and temperature distribution is quantified for a single bubble impacting and bouncing, with the results explained in terms of the bubble dynamics and the surrounding fluid motion.

\section{Experimental Apparatus}

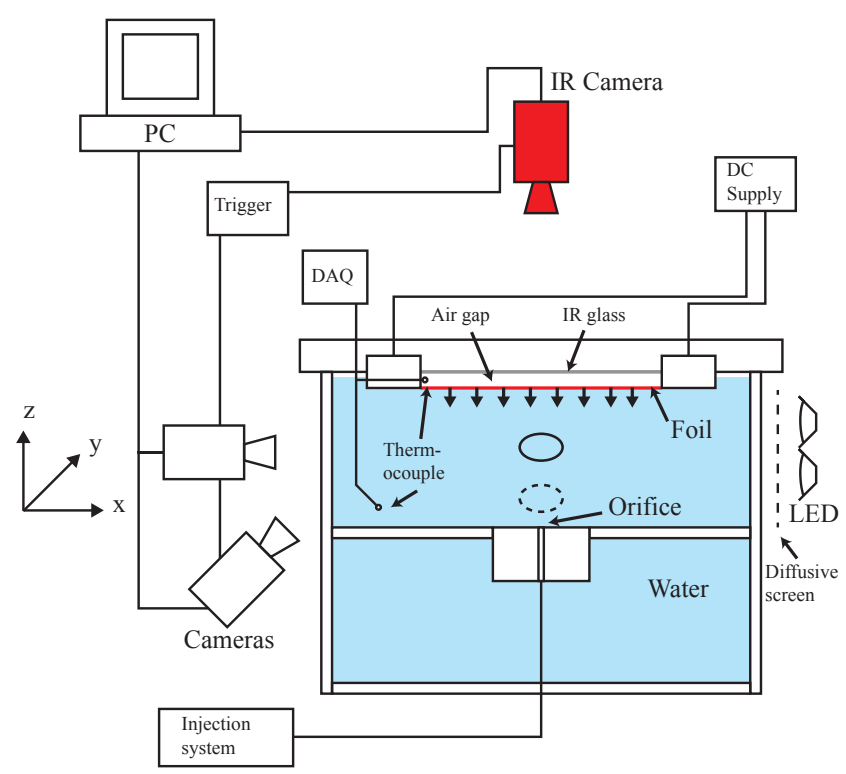

Fig. 1 Experimental set-up. The High-speed cameras are orientated perpendicular to one another, with the IR camera being placed above the heated surface. The LED are placed behind a diffusive screen, opposite to the camera position, illuminating the bubble from behind. 
The experimental apparatus is shown in Fig. 1. It consists of a $110 \times 95 \times 195 \mathrm{~mm}^{3}$ tank constructed from $3 \mathrm{~mm}$ thick glass with a horizontally mounted test surface placed at the top. The tank contains an adjustable bubble injection orifice, a controllable heated surface and a bulk water thermocouple. A high-speed infrared camera and two CCD cameras are mounted directly above and horizontally at the sides of the test section respectively. To capture rapid movement of the bubble as it rises, high intensity lighting is required to enhance the outline of the bubble. This is provided by three high intensity light emitting diode (LED) strips mounted opposite each camera. A diffusive screen is placed between the lights and glass tank to create a uniform light sheet. Two NAC Hi-Dcam II digital high-speed colour cameras are used in these experiments. Both high speed cameras give a spatial resolution of approximately 37 $\mu \mathrm{m} /$ pixel.

A FLIR SC6000 high resolution, high speed infrared camera is used to capture spatial and temporal changes in the surface temperature during bubble impingement events. The camera has a $640 \times 512$ pixel focal plane array and an InSb 3-5 $\mu m$ sensor which is vacuum sealed within the cooler assembly. For the present study, the spatial resolution of the IR camera was $203 \mu \mathrm{m} /$ pixel. All three cameras are set to record at a frequency of $1000 \mathrm{~Hz}$ and are triggered simultaneously using a $5 \mathrm{~V}$ TTL signal. The IR camera has an exposure time of 0.8 $m s$, while each high speed camera has an exposure time of $0.5 \mathrm{~ms}$.

The bubble injection system is mounted on a movable platform, which allows the injection point to be adjusted to varying distances from the test surface. For the present study the distance was $30 \mathrm{~mm}$. The injection orifice is a $2 \mathrm{~mm}$ hole machined into a stainless steel base which is connected to a Hamilton GASTIGHT $10022.5 \mathrm{ml}$ syringe by means of a $300 \mathrm{~mm}$ length of tube with a $0.5 \mathrm{~mm}$ internal diameter. An accurate bubble injection rate is achieved by using a KD Scientific model 220 series infusion pump set to an injection flowrate of $100 \mathrm{ml} / \mathrm{hr}$ for the present study. The growth rate of the bubble was found to be linear, with the stepper motor having no influence on the bubble growth dynamics as shown by Albadawi et al. [26-28]. The tank is filled with ultra-pure water, purified with a Millipore purification system. The water temperature was approximately $19.7 \pm 1^{\circ} \mathrm{C}$.

The test surface consists of a metal foil, the lower side of which is in contact with the water while the upper side is insulated by means of infrared transparent glass. The test surface is a $10 \mu m$ thick Constantan Alloy Cu55/Ni45 rolled foil supplied by Goodfellow Ltd. The foil is clamped between two busbars using silver
Table 1 Foil and paint properties.

\begin{tabular}{|l|l|}
\hline Density $\rho_{f}$ & $8637 \mathrm{~kg} / \mathrm{m}^{3}$ \\
Thermal conductivity $k_{f}$ & $23.13 \mathrm{~W} / \mathrm{mK}$ \\
Specific heat $C_{f}$ & $401.5 \mathrm{~J} / \mathrm{kgK}$ \\
Density $\rho_{p}$ & $1277 \mathrm{~kg} / \mathrm{m}^{3}$ \\
Thermal conductivity $k_{p}$ & $0.095 \mathrm{~W} / \mathrm{mK}$ \\
Specific heat $C_{p}$ & $2835 \mathrm{~J} / \mathrm{kgK}$ \\
Thermal diffusivity $\alpha_{p}$ & $2.62 \times 10^{-8} \mathrm{~m}^{2} / \mathrm{s}[31,32]$ \\
\hline
\end{tabular}

based electrically conductive epoxy. The top side of the foil viewed by the IR camera is coated with matt black paint with an emissivity of 0.95 . To ensure an accurate calibration the procedure is performed with the glass window in place. This in situ calibration process, as used by Schulz [29] and Ochs et al. [30] ensures that the effect of the infrared window and paint emissivity are accounted for. The paint layer thickness was determined to be $11.62 \pm 0.87 \mu \mathrm{m}$. The foil and paint properties are shown in Table 1.

The foil is insulated on the upper surface by a $3 \mathrm{~mm}$ air gap. The air gap is maintained by a Calcium Fluoride (CaF2), $1 \mathrm{~mm}$ thick IR transparent viewing window. This polished glass window has a very high percentage transmissibility, approximately $95 \%$ for a depth of $1 \mathrm{~mm}$.

In order to evaluate the convective heat flux, a pixelby-pixel energy balance is applied to the heater. Eq. 1 accounts for generated heat flux ( $q_{\text {gen }}^{\prime \prime}$, defined in Eq. 2 ), while $q_{\text {cond }}^{\prime \prime}$ is the heat conducted through the $3 \mathrm{~mm}$ air layer, to the $\mathrm{CaF}_{2}$ glass. The air within the gap is assumed to be stagnant, as the Rayleigh number for the cavity was estimated to be $R a=47$. This compares with a critical value of $R a_{c}=1708$, as reported by Incropera et al. [33]. Therefore, heat transfer from the bottom to the top surface occurs by conduction and radiation. $q_{r a d}^{\prime \prime}$ is the radiation from the rear side of the foil; there is very little radiation from the front of the foil, both due to the foil's low emissivity and the fact that the adjacent water has low transmissivity in the wavelength range considered. Lateral heat conduction and heat storage within the foil are also accounted for in Eq. 1, which is solved by applying a second order central-difference approximation to the spatial derivatives and a first order central-difference approximation to the time derivative [34-36]. Results in this investigation are discussed in terms of the parameters defined as follows: 

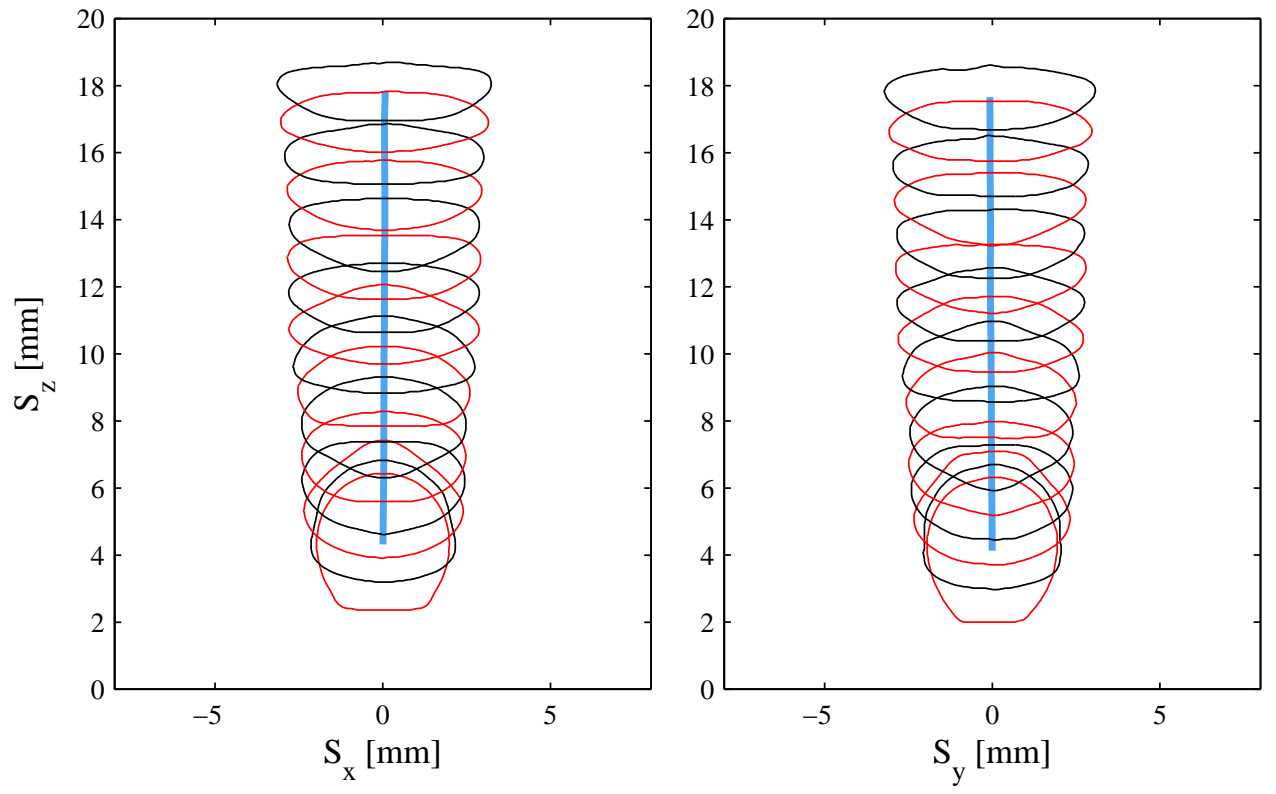

Fig. 2 Sequence depicting the path and shape of a $4.1 \mathrm{~mm}$ bubble released from a height of $20 \mathrm{~mm}$. Both the $\mathrm{x}-\mathrm{z}$ and $\mathrm{y}-$ $\mathrm{z}$ planes at time intervals of $4 \mathrm{~ms}$ between red and black outlines for a bubble.

$q_{\text {conv }}^{\prime \prime}=q_{\text {gen }}^{\prime \prime}-q_{\text {cond }}^{\prime \prime}-q_{\text {rad }}^{\prime \prime}+$

$\left(k_{f} \delta_{f}+k_{p} \delta_{p}\right)\left(\frac{\partial^{2} T}{\partial x^{2}}+\frac{\partial^{2} T}{\partial y^{2}}\right)-$

$$
\left(\rho_{f} C p_{f} \delta_{f}+\rho_{p} C p_{p} \delta_{p}\right) \frac{\partial T}{\partial t}
$$

$q_{g e n}^{\prime \prime}=\frac{I_{\text {elec }}^{2} R_{\text {elec }}}{d x^{2}}$

$R_{e l e c}=\rho_{\text {elec }} \frac{l}{A_{c s}}$

where $I_{\text {elec }}$ is the current through one foil element of resistance, $R_{\text {elec }} \cdot \rho_{\text {elec }}$ is the electrical resistivity of Constantan, $l$ is the length parallel to the current flow and $A_{c s}$ is the cross-sectional area of the foil. A dimensionless temperature difference, $T_{m}$, is defined as:

$T_{m}=\frac{T_{s, t=n}-T_{\infty}}{T_{s, t=0}-T_{\infty}}$

where $T_{s, t=n}$ is the surface temperature at each time step, from $t=0$ to $t=8 \mathrm{~s}, T_{s, t=0}$ is the surface temperature at the beginning of the test and $T_{\infty}$ is the bulk fluid temperature. Increases in $T_{m}$ are indicative of decreased heat transfer whereas decreasing $T_{m}$ is indicative of increased local heat transfer.
The uncertainty in the convective heat flux due to the motion of the bubble and ensuing wake is evaluated utilizing an error propagation analysis on Eq. 1 as described by Donoghue et al. [25]. The resulting uncertainty in the measured heat flux ranges from 3.5\%$16.6 \%$, with the maximum being at the highest convective heat flux level due to the additional error associated with the lateral conduction and thermal storage terms.

\section{Results and Discussion}

\subsection{Bubble Motion}

Once the bubble has departed the injection orifice it quickly gains momentum as buoyancy forces accelerate it upward towards its terminal velocity. In this case the bubble has an equivalent diameter of $d_{e}=4.1 \mathrm{~mm}$, which was determined by equating the volume of the bubble immediately after detachment from the orifice to that of a sphere. The bubble's terminal velocity is dependent on the Morton, Reynolds and Eötvös numbers with it, according to Clift et al. [37], expected to be approximately $250 \mathrm{~mm} / \mathrm{s}$. In this case due to the confinement, a stable terminal velocity was never achieved as the orifice to impact surface distance was too low but a maximum oscillatory velocity of $287 \mathrm{~mm} / \mathrm{s}$ was achieved prior to impact. The maximum oscillatory velocity is the peak velocity due to the sinusoidal motion, induced due to the change in the bubble's aspect ratio. 
(a)

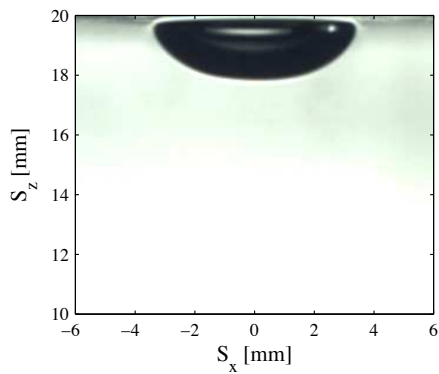

(d)

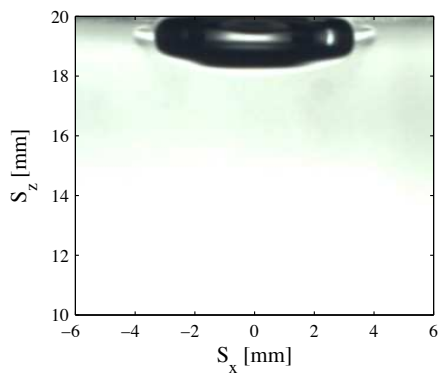

(g)

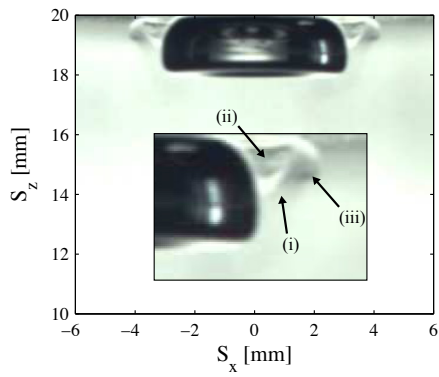

(j)

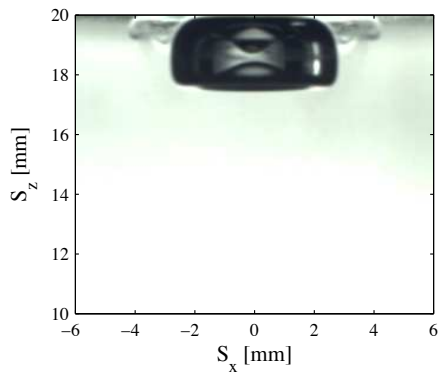

(m)

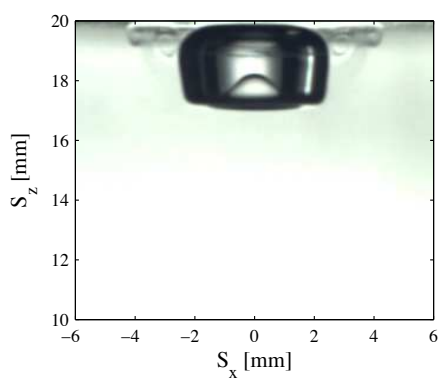

(b)

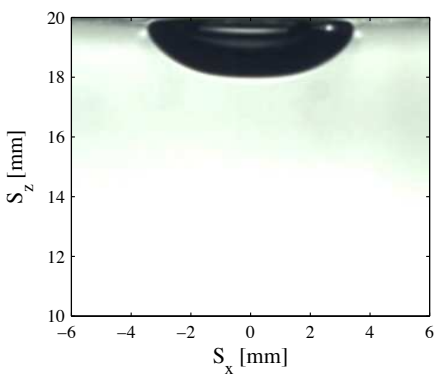

(e)

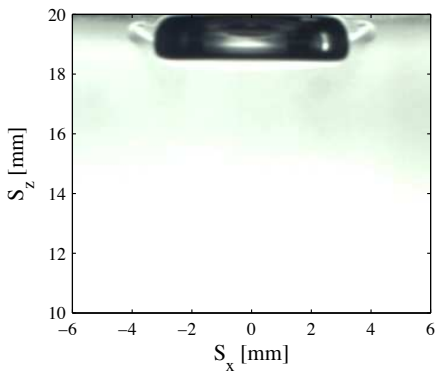

(h)

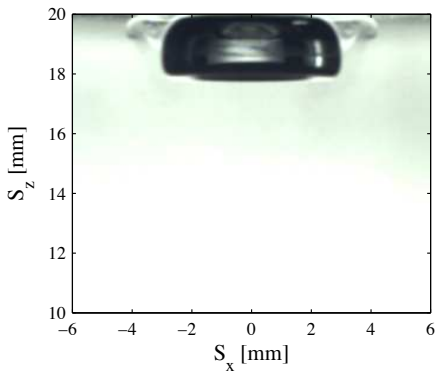

(k)

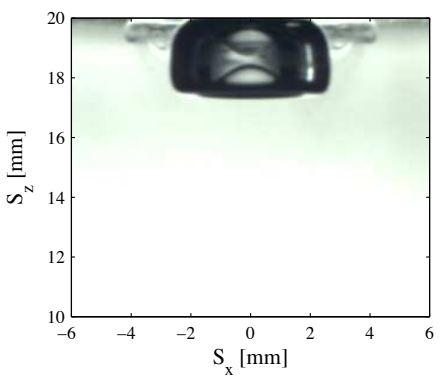

(n)

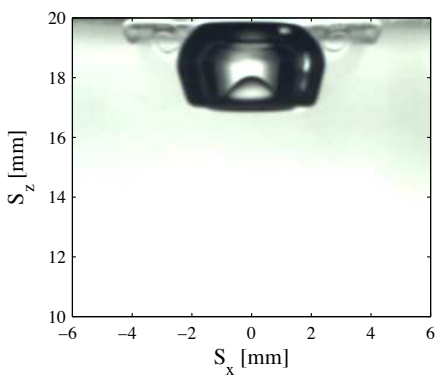

(c)

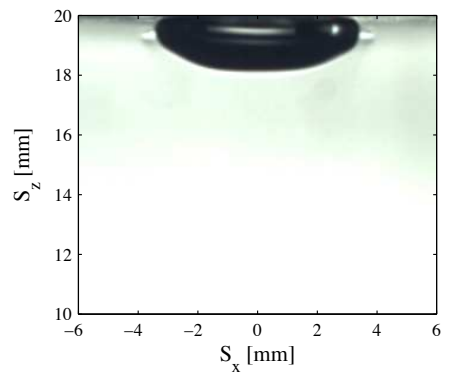

(f)

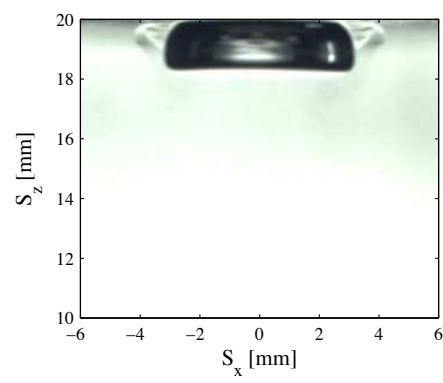

(i)

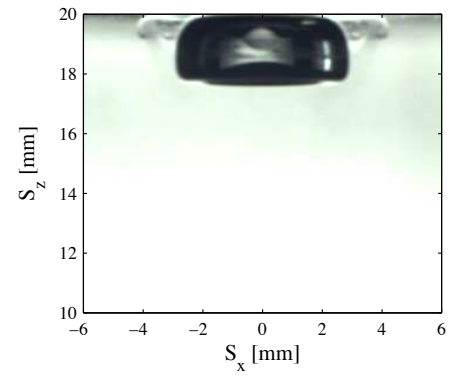

(l)

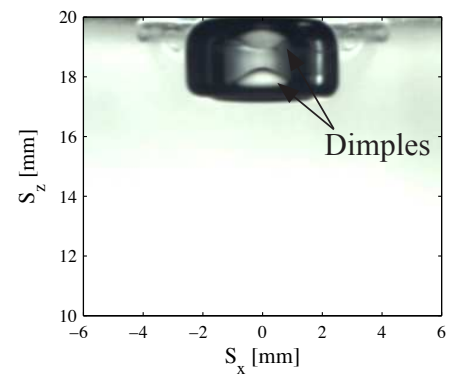

(o)

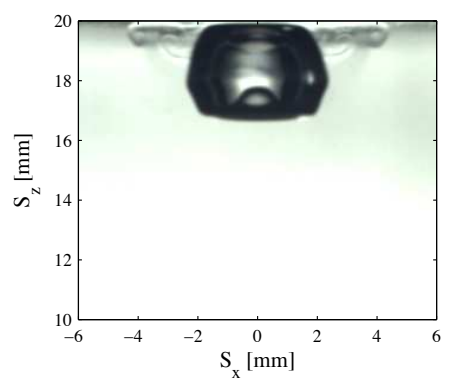

Fig. 3 Sequence depicting the path and shape of a $4.1 \mathrm{~mm}$ bubble released from a height of $20 \mathrm{~mm}$ during the initial impact $(0 \mathrm{~ms})$ and rebound. The time between images is $1 \mathrm{~ms}$. (g) illustrates the following (i) dark fluid, (ii) inner vortex and (iii) outer vortex.

Larger bubbles display more complex rise patterns when compared to bubbles with equivalent diameters of less than $1.5 \mathrm{~mm}$; these include both zig-zag and spiral rise paths, or a combination of both patterns. As noted by previous literature, the rise path of larger bubbles is dictated largely by the previously mentioned dimensionless numbers. However, as noted by Tomiyama et al. [38] and Wu \& Gharib [39] the path, shape and veloc- 


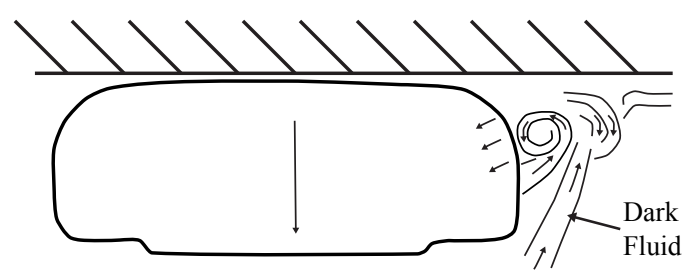

Fig. 4 Bubble outline and approximate fluid motion at a time of $7 \mathrm{~ms}$, corresponding to Fig 3 (g).

ity of the bubble are extremely sensitive to initial shape deformation during the departure process. Thereby, the injection process will have a profound effect on the bubble's shape and motion. Note that Marangoni convection has not been observed during bubble impact due to the order of magnitude of the bubble's velocity when compared to that expected for Marangoni convection to occur. The absence of this type of flow has also been confirmed by means of PIV measurements, i.e. when the bubble is attached Marangoni flow would be visible as a toroidal vortex around the bubble.

Fig.2 illustrates the rise path of a $4.1 \mathrm{~mm}$ bubble released from a nozzle-to-target height of $20 \mathrm{~mm}$ from a $2 \mathrm{~mm}$ orifice. Due to the low rise height the bubble rose vertically. As the bubble approached the solid boundary it began to decelerate before impacting the surface, with the drainage of the liquid film being described using lubrication theory [40]. During this period a number of forces act to slow the advance of the bubble, which include the added mass due to the acceleration of the liquid around the bubble, the history force, which accounts for the finite time required by the surrounding flow to adjust itself to the change in slip velocity (Klaseboer et al. [41]), the lift force due to the vorticity in the flow and the buoyancy and drag forces. When the bubble approaches the wall, the film pressure begins to increase, the corresponding force becoming much larger in magnitude than the buoyancy force, as noted by Klaseboer et al. [41].

As previously reported by Donoghue et al. [25], if a bubble impacts a horizontal surface at an angle, the ensuing wake impacts and develops in the direction away from the bubble. This holds true for all bubble sizes tested if the impact is at a significant angle, which causes path deviation. The amount of path deviation and the variation in bubble size will dictate the area affected by the bubble and its ensuing wake. In cases where a bubble rises vertically, with the bubble's major axis being approximately parallel to the heated surface, the enhancement due to the ensuing wake is found to be approximately annular. This type of convective enhancement pattern is found to occur more for larger bubbles whose rise path is less susceptible to deviation.

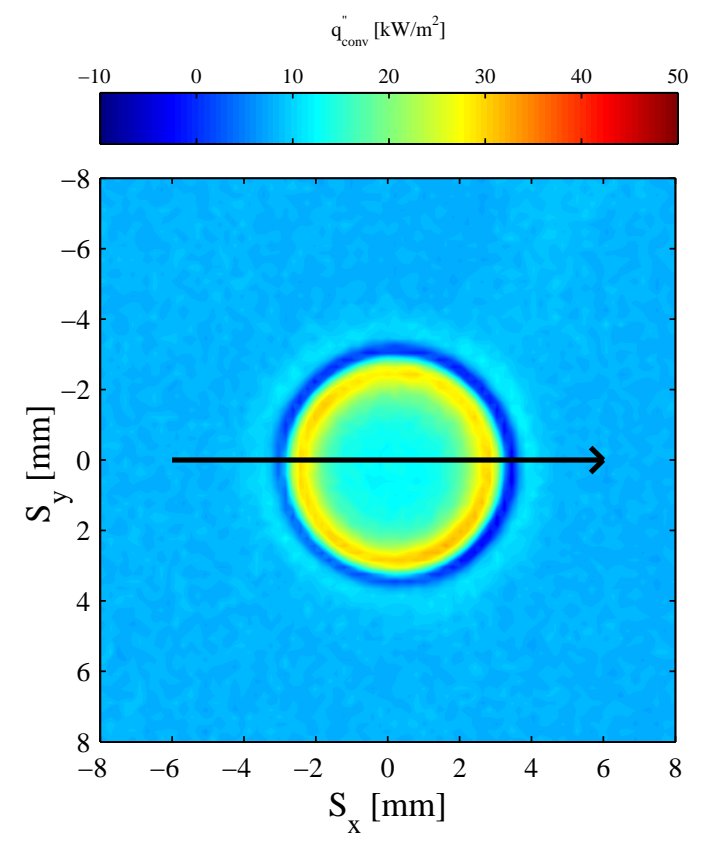

(a)
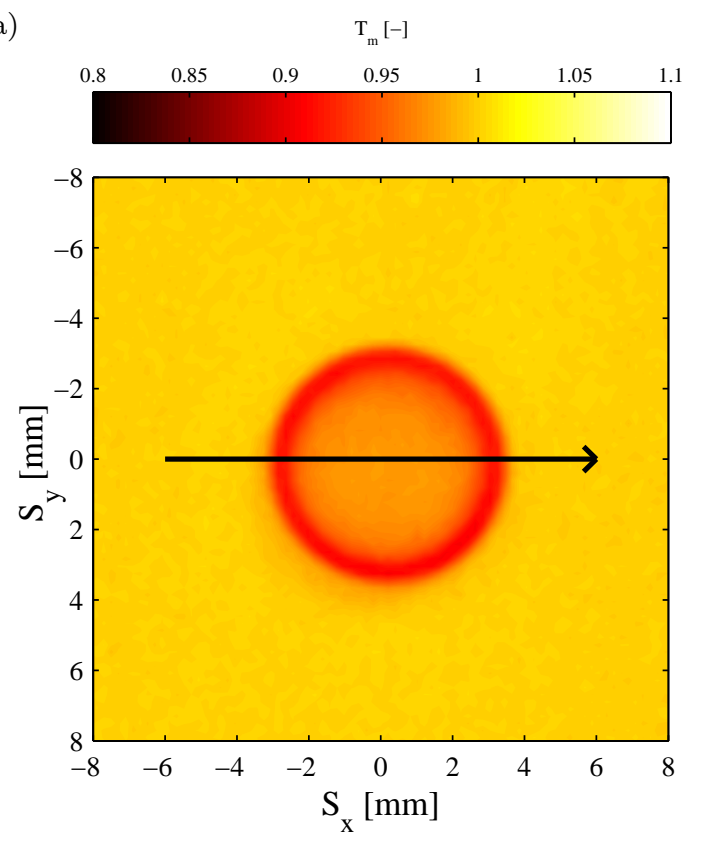

(b)

Fig. 5 (a) Convective heat flux and (b) dimensionless surface temperature for a $4.1 \mathrm{~mm}$ bubble, at a time of $10 \mathrm{~ms}$ after the initial impact. The black line indicates the slice shown in Fig. 6.

Fig. 3 illustrates the initial rebound of a $4.1 \mathrm{~mm}$ bubble, released from a height of $20 \mathrm{~mm}$, between the times of $0-14 \mathrm{~ms}$ post impact, with $0 \mathrm{~ms}$ being the moment of impact. These images are of interest due to the observable fluid temperature gradients, as a result of reduced lighting, and allow partial insight into the fluid motion during the initial impact and rebound 
event. Both the bubble and the subsequent wake impact were found to be symmetric and approximately central in the test section. The variation in the fluid density, which is coupled to the wake impact, is evident at either side of the bubble in Fig. 3 .

In Fig. 3 at a time of $1 \mathrm{~ms}$ after impact, a region of counter clockwise fluid motion occurs on the right hand side of the bubble at a position of $S_{x}=4 \mathrm{~mm}$, with the opposite occurring on the left hand side. This rotation of fluid was found to be linked to the bubble's rebound. Between times of $2-3 \mathrm{~ms}$ (Fig. $3 c \& d$ ) this vortex grows slightly, extending beyond $S_{x}=4 \mathrm{~mm}$ as the bubble pulls inwards. Fig. $3 e$ and $f$ illustrate the bubble motion at 4 and $5 \mathrm{~ms}$; in this case a secondary, inner vortex appears due to the continued retraction of the bubble. The direction of this new inner vortex is again counter clockwise on the bubble's right hand side, with the outer vortex being pushed towards the surface and now rotating in a clockwise direction. The inner vortex was determined to grow in size between times of 4-14 ms, which may be inferred from Fig. $3 e$ to $o$. At times of $10-13 \mathrm{~ms}$, just visible in Fig. $3 k$ to $n$, at a position of $S_{x}=3 \mathrm{~mm}$, a region of "dark" fluid visually appears at the edge of bubble, ending between the inner and outer vortices, which is depicted in Fig. 3 $g$ and illustrated in Fig. 4. This stream of fluid actually begins earlier, but is difficult to illustrate in the still photographs, though can be identified from the video footage. It was found to initiate at the outer corner of the bubble, the point furthest from the surface, and is thought to be the stream of fluid which reverses the direction of the original outer vortex.

\subsection{Local Heat Transfer}

Fig. 5 illustrates the convective heat flux and dimensionless surface temperature at a time of $10 \mathrm{~ms}$ for the $4.1 \mathrm{~mm}$ bubble impacting from a height of $20 \mathrm{~mm}$. The direction and location of a surface line is shown, intersecting the centre of the impact zone, which relates to the data shown in Fig. 6 \& Fig. 7. Once again the symmetry of the cross section is noteworthy. In this case the approximate surface temperature prior to the bubble's impact was $41.8 \pm 0.3^{\circ} \mathrm{C}$

In Fig. 6, for the initial period following impact, two different enhancement zones are present; the first is due to the bubble's impact, with the second being assumed to be a result of the wake. As the bubble impact and developing wake is symmetric, only one side needs to be commented on. The effect of the bubble impact and the beginning of its rebound is evident between the limits of $S_{s l}=1.5-3 \mathrm{~mm}$. The initial impact edge of the bubble is at $S_{s l}=3 \mathrm{~mm}$; as the release height is low the enhancement is insignificant until a time of 4 - $6 \mathrm{~ms}$. The initial impact convective heat flux has a maximum of approximately $40 \mathrm{~kW} / \mathrm{m}^{2}$ at $6 \mathrm{~ms}$. As the edges of the bubble pull inwards this ring of convective enhancement shifts inwards, being at a position of $S_{s l}=1.5 \mathrm{~mm}$ at a time of $20 \mathrm{~ms}$. Notably, as the edges of the bubble move inwards, the region where the initial impact occurred experiences a reduction in convective heat flux as the surface temperature rises. This region of low convective heat flux is thought to be related to the counter clockwise inner vortex, visible in Fig. $3 j$. From Fig. 5, the outer ring of negative convective heat flux at a time of $10 \mathrm{~ms}$ is evident, as the bubble begins to recover its shape.

At the outer regions $(3-4.5 \mathrm{~mm})$ of enhancement in Fig. 6, the impact of the wake can be inferred to begin at a time of $12 \mathrm{~ms}$. This is related to the flow field shown in Fig. $3 \mathrm{~m}$, whereby the "dark" cool fluid is forced to flow between the inner and outer vortices. The outer initial vortex appears not to enhance or reduce heat transfer during the initial impact and rebound, since this vortex is slightly separated from the surface. Once the ensuing wake (dark fluid) impacts the surface, the convective heat flux increases significantly, to levels just above $150 \mathrm{~kW} / \mathrm{m}^{2}$, in a short time period. The peak in enhancement in Fig. 6 occurs between the times of 16 $18 \mathrm{~ms}$, while at a time of $20 \mathrm{~ms}$, the peak is depressed.

Fig. 7 illustrates the convective heat flux and dimensionless surface temperature between times of $20-40$ $m s$. The bubble was found to completely rebound from the surface at a time of $23 \mathrm{~ms}$ as illustrated in Fig. 8; again the bubble's rebound process was found to be symmetric. During the time period shown in Fig. 7, after $23 \mathrm{~ms}$ the bubble is away from the surface. In this case, the impacted wake was found to spread beyond $\pm 4 \mathrm{~mm}$; instead, it appears that the wake shifts inwards, as far as $\pm 2 \mathrm{~mm}$. This rapid shift inwards is due to the bubble's shape change, pulling a portion of the outward moving wake inwards to $\pm 2 \mathrm{~mm}$. This shift is apparent in Fig. 7 between the times of 20 and 40 $m s$. Thus, the bubble originally extended as far as \pm 3.5 $\mathrm{mm}$ (Fig. $3 \mathrm{a}$ ), which is reduced to $\pm 2 \mathrm{~mm}$ (Fig. 8). Notably, the enhancement zone does not extend inside the extremities of the bubble, which in this case is \pm 2 $m m$.

This wake motion results from the shape of the bubble prior to leaving the surface, together with the influence of the fluid which is trapped within the impact dimple. The outline of the bubble at two time periods of 14 and $19 \mathrm{~ms}$ is sketched in Fig. 9. In Fig. 9 a, both the inner and outer vortex are present, as inferred from the temperature gradients. As the bubble has retracted from its impact shape, the lower edges pull inwards and 

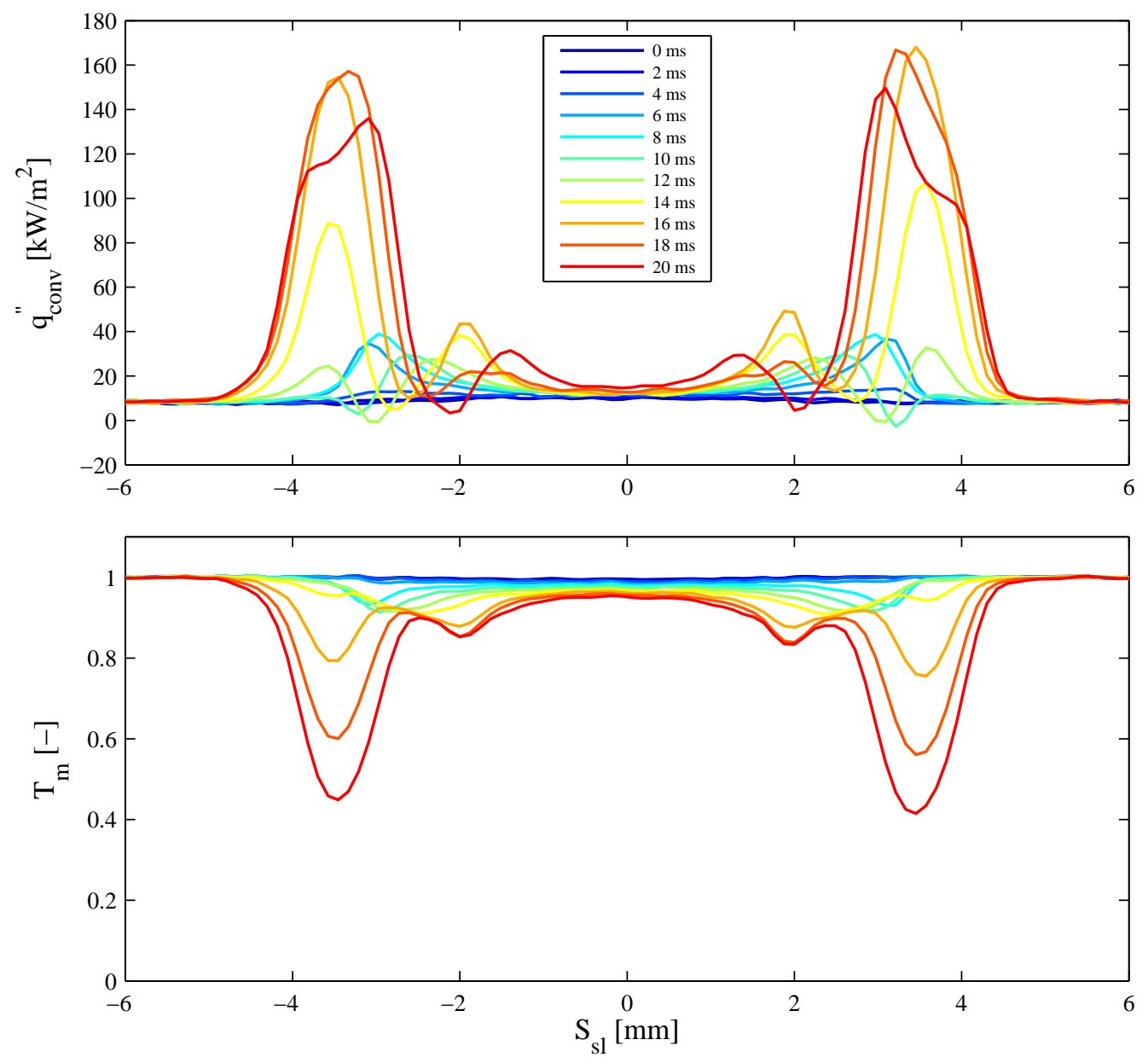

Fig. 6 Convective heat flux and dimensionless surface temperature along a defined line on the surface, with a $\Delta t=2 m s$, during a time period of $0-20 \mathrm{~ms}$. The release height is $20 \mathrm{~mm}$.

this drags the inner vortex inwards. In this case the rising wake continues its rise path around the bubble as depicted.

Fig. 9 (b) illustrates the bubble at a time of $19 \mathrm{~ms}$. At this instance the bubble has a vertical height of 5 $m m$, with an approximate width of $4 \mathrm{~mm}$ at its widest. As the bubble has pulled inwards, this results in the rising wake, which was impacting in-between the two vortices, being pushed away from the surface. Thus, during the impact process, two dimples form on the bubble's surface: one between the bubble and the surface, the second at the bubble's lower edge. Both dimples can be inferred at a time of $9 \mathrm{~ms}$ in Fig. $3 i$ to $o$. As the bubble continues its retraction, this lower dimple inverts $\left(S_{z}=18 \mathrm{~mm}\right)$, expelling the previously captured wake fluid back against the continually rising wake. This reversal of fluid flow is enhanced by the bubble rebound, which temporarily disconnects the rising wake from the enhancement zone, with the disconnection being illustrated in Fig. $9 a$ and $b$.

Once the bubble has left the surface at a time of 22 $\mathrm{ms}$, the zone extending beyond $\pm 3 \mathrm{~mm}$ experiences a continued temperature reduction, as shown in Fig. 7 . This peak heat flux steadily shifts inwards to $\pm 2 \mathrm{~mm}$ at a time of $40 \mathrm{~ms}$, all the while steadily reducing due to the change in temperature. The source of this enhancement has been inferred from a single PIV test in which the bubble bounced in a similar fashion to that shown here [42]. Between the time period of $20-40$ $m s$, the outer vortex on the right hand side rotates in a clockwise direction; this rotation moves fluid towards the void left by the bubble's shape recovery. In Fig. 8 $b$, the vortex is located at approximately $\pm 4 \mathrm{~mm}$ at a height of $19 \mathrm{~mm}$, while the "void" is located between the bubble and the rolling vortex. This vortex motion (clockwise) combines with the motion of the bubble to 

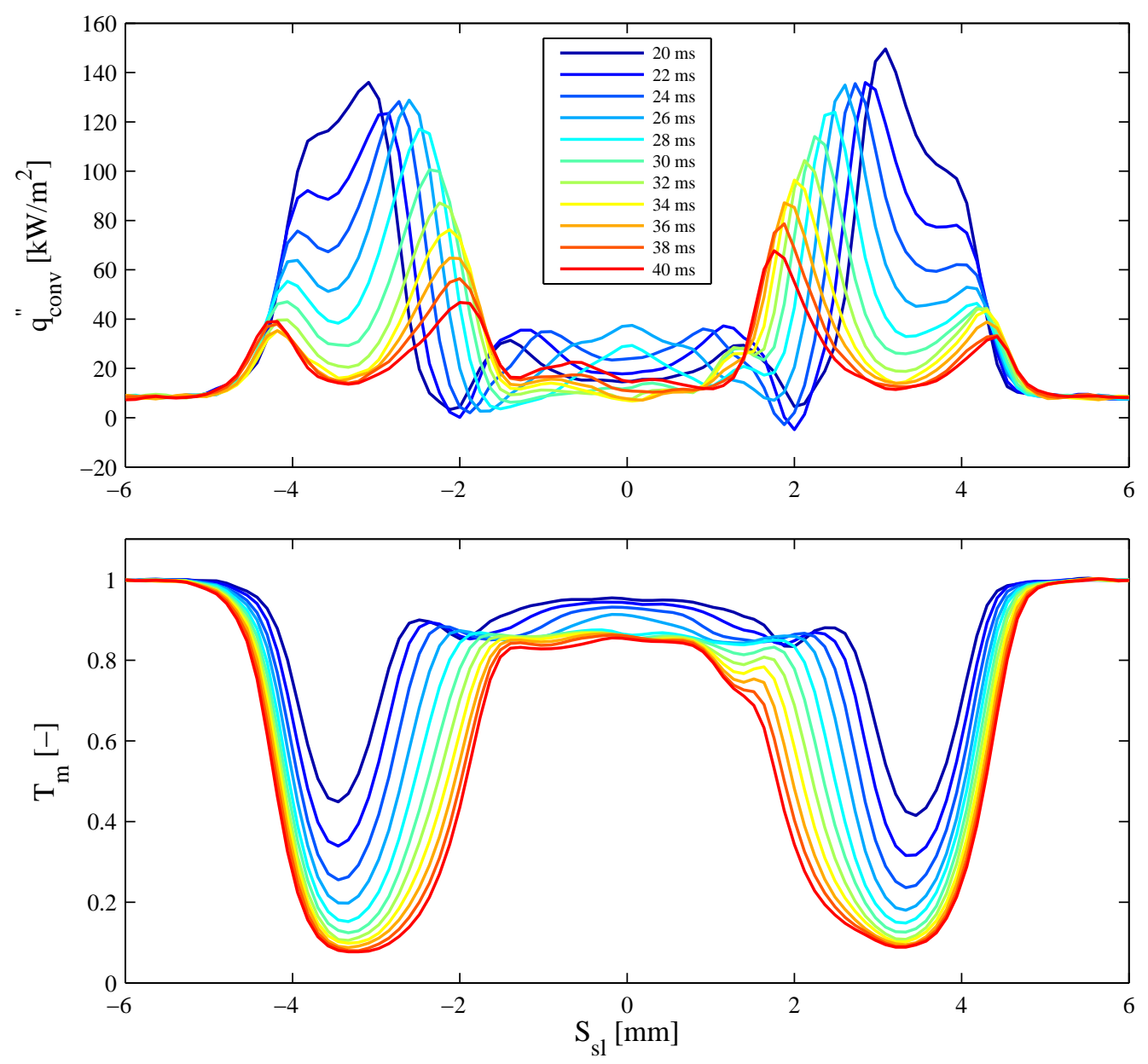

Fig. 7 Convective heat flux and dimensionless surface temperature along a define slice in the surface, with a $\Delta t=2 m s$, during a time period of $20-40 \mathrm{~ms}$. The release height is $20 \mathrm{~mm}$.

cool the surface, by circulating fluid into the void. The fluid which is pulled inwards is the bulk fluid which is adjacent to the boundary layer, now a mixture of both warm and cool fluid. The extent of the wake is visible in Fig. 10 at a time of $30 \mathrm{~ms}$.

The bubble's dimple was found to be fully inverted at a time of $21 \mathrm{~ms}$, resulting in both increases and decreases in convective heat flux which are observable within the limits of $\pm 2 \mathrm{~mm}$ in Fig. 7, while the main portion of the wake does not encroach inside this limit. This limit again was found to be the minimum width of the bubble upon rebound from the surface.

At a time of $40 \mathrm{~ms}$ as seen from Fig. 7, the convective heat flux has reduced significantly. As inferred from PIV images [42], this is due to the vortex shifting further outwards, reducing in strength, and due to the fact that the bubble is no longer pulling inwards. This lower enhancement may also be linked to the inversion of the bubble's impact dimple, which is thought to contain both warm boundary layer fluid and cool bulk fluid.

Fig. 11 illustrates the variation in the convective heat flux and dimensionless surface temperature between the times of $40-80 \mathrm{~ms}$ for the $4.1 \mathrm{~mm}$ bubble and a $20 \mathrm{~mm}$ release height. During this time period the bubble re-impacts the surface at a time of $48 \mathrm{~ms}$ before rebounding from the surface again at a time of $70 \mathrm{~ms}$. The initial re-impact at $48 \mathrm{~ms}$ causes an increase in the convective heat flux in the central region, while at the bubble's edge negative heat flux occurs. This ring of negative heat flux is evident in Fig. $12 a$ at a time of 50 $m s$. This re-impact causes both enhancement and reduction of the convective heat transfer, particularly at a position of $\pm 2 \mathrm{~mm}$. At this position, there is initially positive enhancement of approximately $40 \mathrm{~kW} / \mathrm{m}^{2}$; as the bubble re-impacts the surface, the edges of the bubble impact this region with a greater velocity than the 


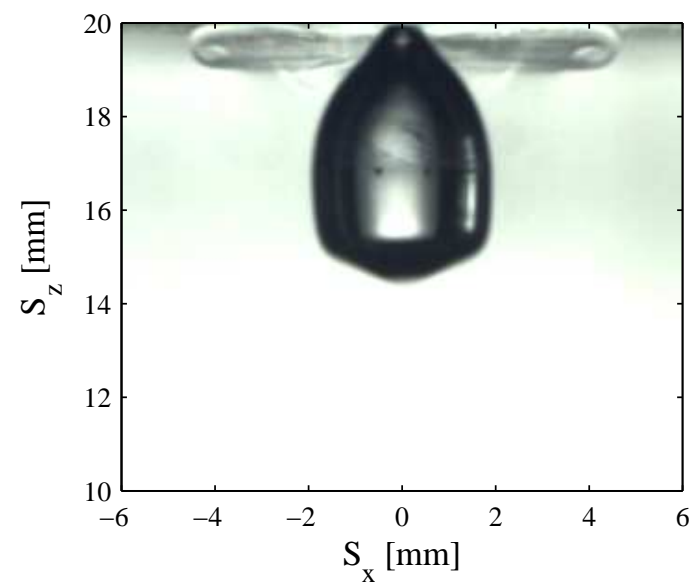

(a)

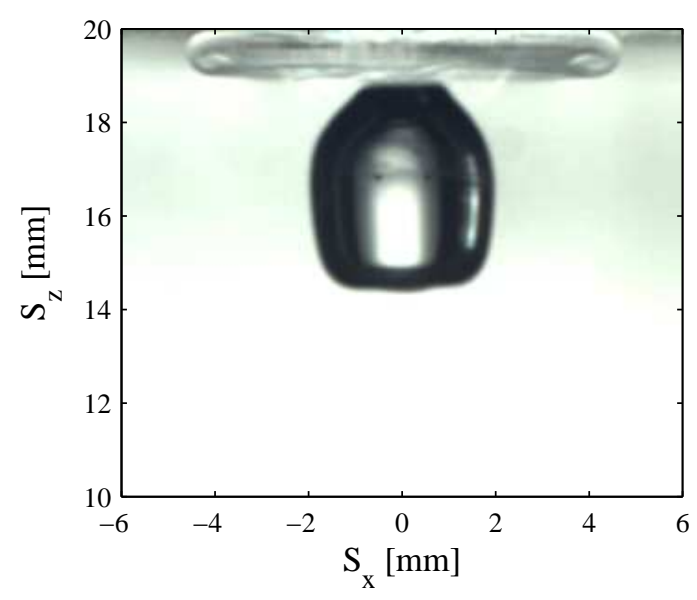

(b)

Fig. 8 Impact of a $4.1 \mathrm{~mm}$ bubble released from a height of $20 \mathrm{~mm}$, (a) at a time of $21 \mathrm{~ms}$ and (b) $23 \mathrm{~ms}$.

main portion of the bubble. This causes the rapid decrease in convective heat flux at this point, as warm fluid is forced against the lower temperature surface, to an approximate value of $-35 \mathrm{~kW} / \mathrm{m}^{2}$. As the bubble rebounds from the surface, this region experiences positive enhancement of approximately $30 \mathrm{~kW} / \mathrm{m}^{2}$, which steadily reduces over time. The bubble re-impacted the surface once more at a time of $94 \mathrm{~ms}$ (not shown), which was again found to affect the convective enhancement. During this time period the outer vortex, $S_{s l}>4 \mathrm{~mm}$, cools the surface slowly, with the vertical extent of the vortex increasing in size to around $2.5 \mathrm{~mm}$. Eames \& Dalziel [43] investigated the flow around a rigid sphere (a)

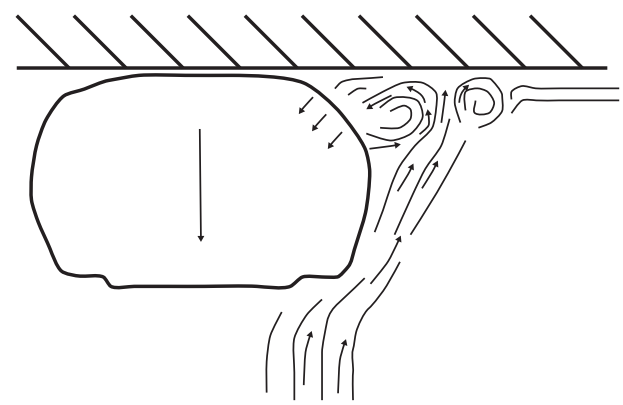

(b)

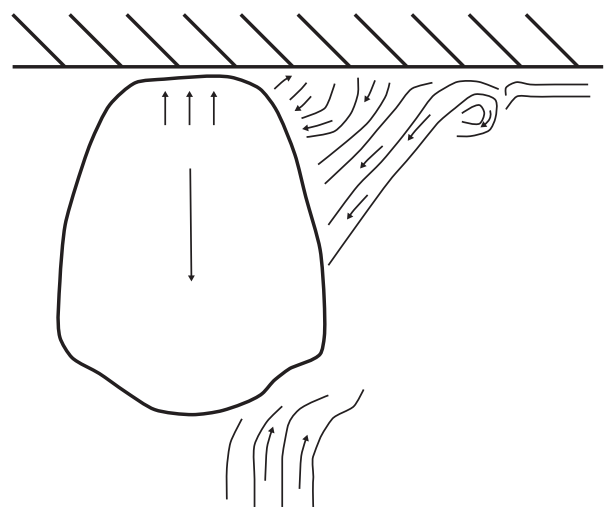

Fig. 9 Bubble outline and approximate fluid motion at a time of (a) $14 \mathrm{~ms}$ and (b) $19 \mathrm{~ms}$.

impacting a solid surface. It was found that when the sphere made contact with the wall, a wake vortex, initially located at the rear of the sphere, was able to shroud the sphere and impact the wall. This generated a secondary vortex ring which, together with the initial wake, was found to push liquid radially outwards. Images captured by Eames \& Dalziel [43] were found to be qualitatively consistent with the results observed in this study with large ellipsoidal bubbles.

\section{Conclusion}

An experimental study has been conducted on a large ellipsoidal bubble impacting a solid horizontal heated surface. Surface temperature variations and resolved convective heat flux are presented, along the bubble motion which were captured at a frequency of $1 \mathrm{kHz}$. The heated surface was an ohmically heated $10 \mu \mathrm{m}$ thick Constantan foil, with a $2 \mathrm{~mm}$ diameter stainless steel orifice being used to generate the $4.1 \mathrm{~mm}$ bubble.

During the bubble's rise, significant wake structures were found to develop at the rear of the bubble, instigated by the change in the bubble's aspect ratio. Variations in aspect ratio and bubble velocity have been linked to the bubble's departure conditions, as shown by Tomiyama et al. [38] and Wu \& Gharib [39]. In cases 
$\mathrm{q}_{\text {conv }}^{\prime \prime}\left[\mathrm{kW} / \mathrm{m}^{2}\right]$

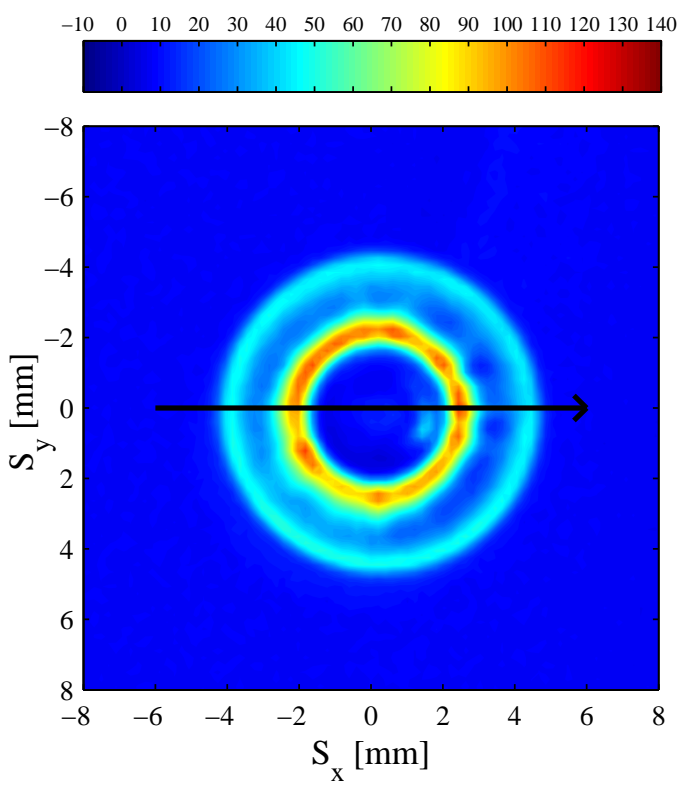

(a)
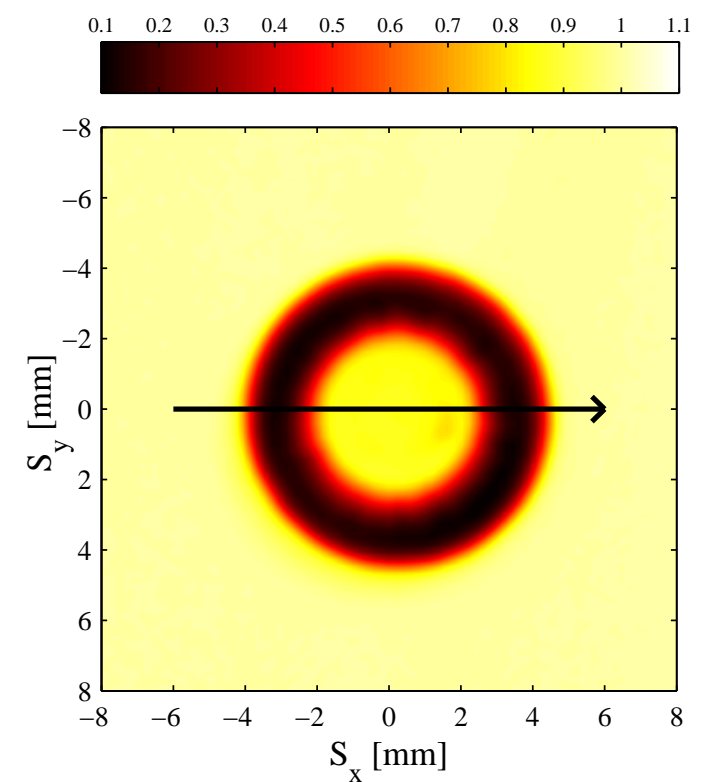

(b)

Fig. 10 (a) Convective heat flux and (b) dimensionless surface temperature for a $4.1 \mathrm{~mm}$ bubble, at a time of $30 \mathrm{~ms}$ after the initial impact. The release height is $20 \mathrm{~mm}$.

where large bubble deformations occur, the wake can become partially separated from the bubble, impacting in separate zones.

In cases where the wake spreads significantly along the surface, a situation can arise in which a rolling vortex which initially cooled the surface becomes counter productive, convecting warm boundary layer fluid on top of the previously cooled surface. If a bubble im- pacts the surface evenly as shown in the present study, the cooling pattern is symmetric, while being split into two regions. The inner region is a result of the bubble impact, while the outer region was found to be due to the ensuing wake moving around the bubble.

This article has provided a detailed review of the bubble impact dynamics and associated heat transfer mechanisms for a single bubble size and rise height. However, a broader based investigation with a wider range of bubble sizes and test conditions is planned as a future study.

Acknowledgements The authors acknowledge the technical support staff of the Mechanical and Manufacturing Engineering Department of Trinity College Dublin. The project is funded by Science Foundation Ireland (SFI) grant number 09-RFP-ENM2151.

\section{References}

1. Y. Yan, D.B.R. Kenning, K. Cornwell, International Journal of Refrigeration 20(8), 583 (1997)

2. G.E. Thorncroft, J.F. Klausner, R. Mei, International Journal of Heat and Mass Transfer 41(23), 3857 (1998)

3. M.A. Atmane, D.B. Murray, International Journal of Heat and Mass Transfer 48(6), 1084 (2005)

4. K. Cornwell, International Journal of Heat and Mass Transfer 33(12), 2579 (1990)

5. D. Qiu, V.K. Dhir, Experimental Thermal and Fluid Science 26(6-7), 605 (2002)

6. Y.M.C. Delauré, V.S.S. Chan, D.B. Murray, Experimental Thermal and Fluid Science 27(8), 911 (2003)

7. B. Donnelly, T.S. O'Donovan, D.B. Murray, Journal of Enhanced Heat Transfer 15(2), 159 (2008)

8. D.B. Donoghue, B. Donnelly, D.B. Murray, Journal of Enhanced Heat Transfer 19(4) (2012)

9. S. Manickam, V. Dhir, International Journal of Heat and Mass Transfer 55(4), 925 (2012)

10. L.S. Fan, K. Tsuchiya, Bubble wake dynamics in liquids and liquid-solid suspensions (Butterworth-Heinemann, Boston, Massachusetts, 1990)

11. C. Brücker, Physics of Fluids 11, 1781 (1999)

12. A.W.G. De Vries, A. Biesheuvel, L. Van Wijngaarden, International Journal of Multiphase Flow 28(11), 1823 (2002)

13. C.H.J. Veldhuis, Leonardo's paradox: Path and shape instabilities of particles and bubbles. Ph.D. thesis, University of Twente, Enschede, University of Twente (2007)

14. T. Sanada, M. Shirota, M. Watanabe, Chemical Engineering Science 62(24), 7264 (2007)

15. H.K. Tsao, D.L. Koch, Physics of Fluids 9, 44 (1997)

16. D. Legendre, C. Daniel, P. Guiraud, Physics of Fluids 17, 097105 (2005)

17. R. Zenit, D. Legendre, Physics of Fluids 21, 083306 (2009)

18. M. Fujasová-Zedníková, L. Vobecká, J. Vejrazka, The Canadian Journal of Chemical Engineering 88(4), 473 (2010)

19. D. Kosior, J. Zawala, K. Malysa, Colloids and Surfaces A: Physicochemical and Engineering Aspects (2012)

20. A. Sato, M. Shirota, T. Sanada, M. Watanabe, Physics of Fluids 23, 013307 (2011) 

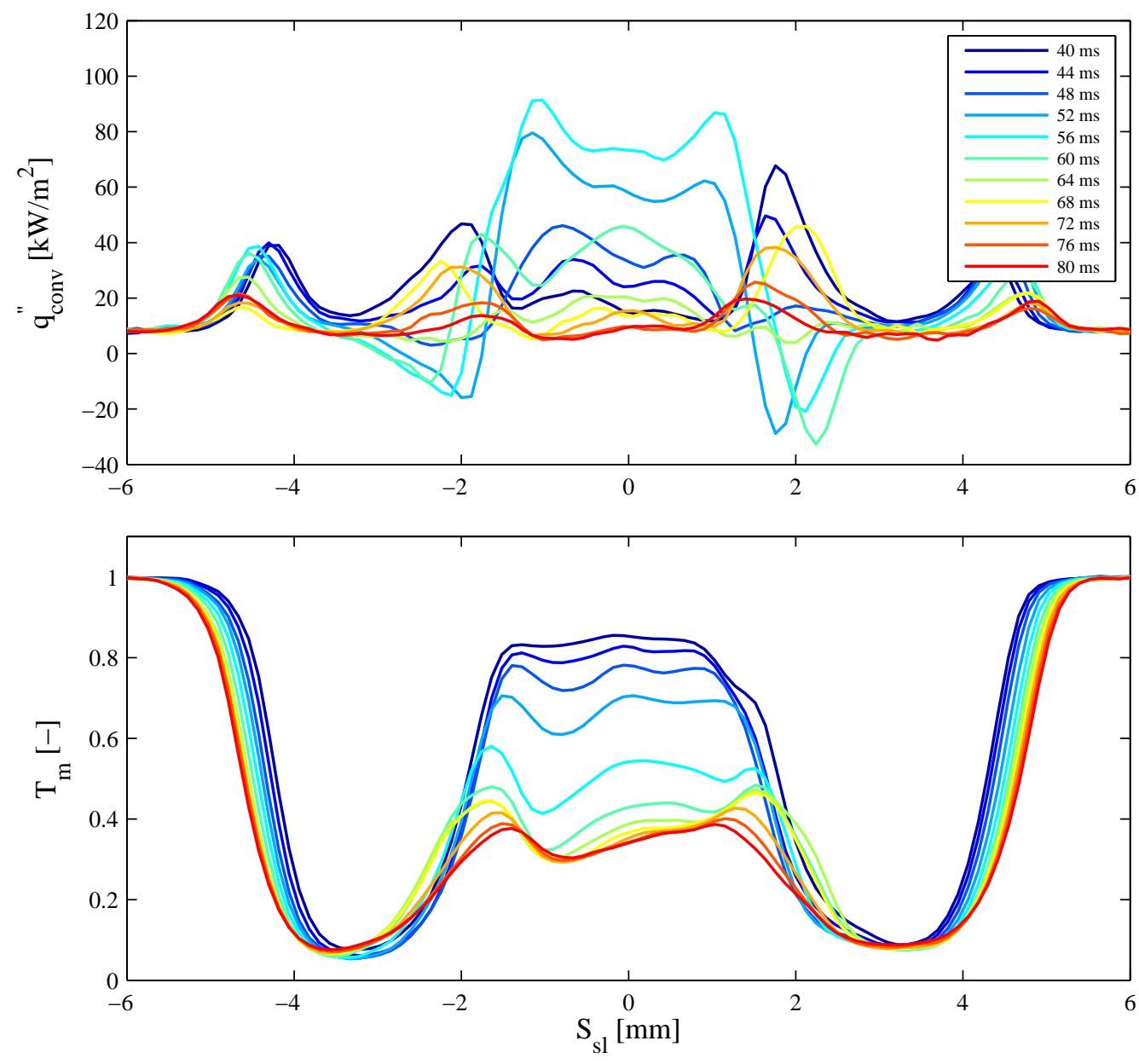

Fig. 11 Convective heat flux and dimensionless surface temperature along a defined slice on the surface, with a $\Delta t=4 m s$, during a time period of $40-80 \mathrm{~ms}$. The release height is $20 \mathrm{~mm}$.

21. D.B. Donoghue, Y.M.C. Delauré, A.J. Robinson, D.B. Murray, in 6th Baltic Heat Transfer Conference (Tampere University of Technology, 2011)

22. B. Donnelly, R. O'Reilly Meehan, K. Nolan, D.B. Murray, International Journal of Heat and Mass Transfer 91, 532 (2015)

23. R. O'Reilly Meehan, B. Donnelly, K. Nolan, D.B. Murray, International Journal of Heat and Mass Transfer 108, $1347(2017)$

24. R. O'Reilly Meehan, B. Donnelly, K. Nolan, T. Persoons, D.B. Murray, International Journal of Multiphase Flow 84, 145 (2016)

25. D.B. Donoghue, A. Albadawi, A.J. Robinson, Y.M.C. Delauré, D.B. Murray, International Journal of Heat and Mass Transfer 71, 439 (2014)

26. A. Albadawi, D.B. Donoghue, A.J. Robinson, D.B. Murray, Y.M.C. Delauré, Chemical Engineering Science 90, 77 (2013)

27. A. Albadawi, D.B. Donoghue, A.J. Robinson, D.B. Murray, Y.M.C. Delauré, International Journal of Multiphase Flow 53, 11 (2013)
28. A. Albadawi, D.B. Donoghue, A.J. Robinson, D.B. Murray, Y.M.C. Delauré, International Journal of Multiphase Flow 65, 82 (2014)

29. A. Schulz, Measurement Science and Technology 11, 948 (2000)

30. M. Ochs, T. Horbach, A. Schulz, R. Koch, H.J. Bauer, Measurement Science and Technology 20 (2009)

31. O. Raghu, J. Philip, Measurement Science and Technology 17, 2945 (2006)

32. J. Philip, M.V. Manjusha, H. Soumya, Review of Scientific Instruments 82(10), 104901 (2011)

33. F.P. Incropera, D.P. DeWitt, T.L. Bergman, A.S. Lavine, Fundamentals of Heat and Mass Transfer, vol. 6 (John Wiley \& Sons, Inc, New York, 2007)

34. J. Stafford, E. Walsh, V. Egan, Measurement Science and Technology 20, 105401 (2009)

35. I. Golobic, J. Petkovsek, M. Baselj, A. Papez, D.B.R Kenning, Heat and mass transfer 45(7), 857 (2009)

36. I. Golobic, J. Petkovsek, D.B.R. Kenning, International Journal of Heat and Mass Transfer 55(4), 1385 (2012)

37. R. Clift, J.R. Grace, M.E. Weber, Bubbles, drops, and particles, vol. 3 (Academic press New York, 1978) 
$\mathrm{q}_{\text {conv }}^{\prime \prime}\left[\mathrm{kW} / \mathrm{m}^{2}\right]$

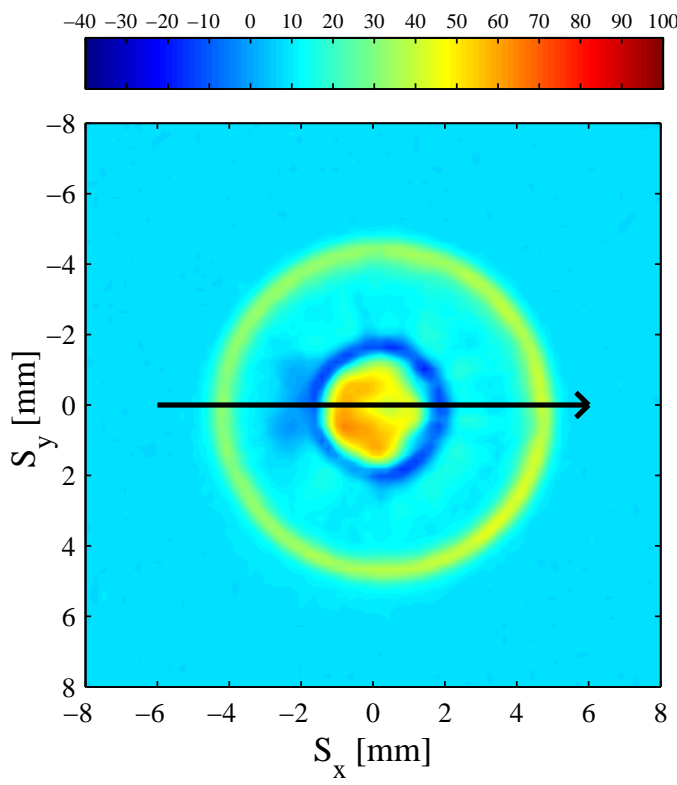

(a)

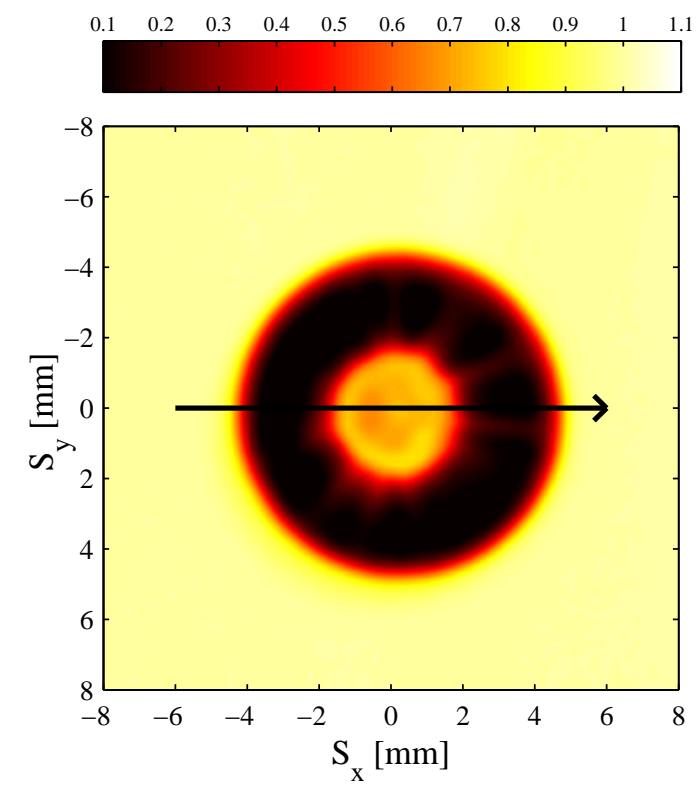

(b)

Fig. 12 (a) Convective heat flux and (b) dimensionless surface temperature for a $4.1 \mathrm{~mm}$ bubble, at a time of $50 \mathrm{~ms}$ after the initial impact. The release height is $20 \mathrm{~mm}$.

38. A. Tomiyama, G.P. Celata, S. Hosokawa, S. Yoshida, International Journal of Multiphase Flow 28(9), 1497 (2002)

39. M. Wu, M. Gharib, Physics of Fluids 14(7), L49 (2002)

40. M.H.W. Hendrix, R. Manica, E. Klaseboer, D.Y.C. Chan, C.D. Ohl, Physical Review Letters 108(24), 247803 (2012)

41. E. Klaseboer, J.P. Chevaillier, A. Maté, O. Masbernat, C. Gourdon, Physics of Fluids 13, 45 (2001)
42. D.B. Donoghue, Bubble impingement and the mechanisms of heat transfer enhancement. Ph.D. thesis, Trinity College (Dublin, Ireland). Department of Mechanical and Manufacturing Engineering, http://hdl.handle.net/2262/77549 (2014)

43. I. Eames, S.B. Dalziel, Journal of Fluid Mechanics 403 $305(2000)$ 